\title{
Editorial
}

\section{Generalized Differential and Integral Equations}

\author{
Rodrigo López Pouso, ${ }^{1}$ Daniel C. Biles, ${ }^{2}$ and Márcia Federson ${ }^{3}$ \\ ${ }^{1}$ University of Santiago de Compostela, 15782 Santiago de Compostela, Spain \\ ${ }^{2}$ Belmont University, Nashville, TN 37212, USA \\ ${ }^{3}$ University of Sao Paulo, Campus de Sao Carlos, 13560-970 Sao Carlos, Brazil \\ Correspondence should be addressed to Rodrigo López Pouso; rodrigo.lopez@usc.es
}

Received 12 December 2013; Accepted 12 December 2013; Published 13 February 2014

Copyright (C) 2014 Rodrigo López Pouso et al. This is an open access article distributed under the Creative Commons Attribution License, which permits unrestricted use, distribution, and reproduction in any medium, provided the original work is properly cited.

The theory of differential equations is always and necessarily "under construction," because more accurate mathematical models usually demand new theoretical developments. For instance, discontinuities or singularities often occur in applications and they are often removed from models just for technical limitations.

On the other hand, the last few years have witnessed an increasing interest in different types of differential and integral equations as mathematical models for real life situations. Besides the classical examples of impulsive equations or equations with deviating arguments, many other types of revisions of the classical concepts of differential or integral equations are being intensively studied: set-valued equations, stochastic equations, fractional equations, fuzzy equations, and many more.

This diversity notwithstanding, it appears that generalized Stieltjes integration provides an unified framework for many of the above types of equations, thus simplifying and improving the theory at the same time.

The special issue succeeded in bringing together a number of papers on many different branches of the theory of differential equations which clearly deserve the adjective "generalized." The editors in charge of this special issue did not expect such a variety when they first proposed a special issue mainly focused on the following topics:

(i) generalized differential and integral equations (such as differential inclusions, stochastic equations, and fractional equations, but not restricted to these three), (iii) fixed point theorems with applications to differential equations,

(iv) generalized integration with applications to differential equations.

While many of papers in the special issue fall inside at least one of the four previous categories, there are some others which do not and yet represent many other interesting topics in this area. For instance, to point out just a few, readers will find in this special issue papers on singular semigroups, fuzzy differential equations, homogeneization of parabolic equations, and interior field methods for Laplace's equation.

For more details on recent and future developments in generalized differential and integral equations, we refer the reader to the survey paper authored by the editors in this special issue.

\section{Acknowledgments}

Finally, the editors wish to express their gratitude to the many authors and reviewers who contributed so greatly to the success of this special issue.

Rodrigo López Pouso Daniel C. Biles

Márcia Federson

(ii) discontinuous or singular equations, 


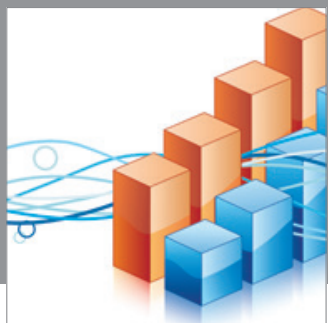

Advances in

Operations Research

mansans

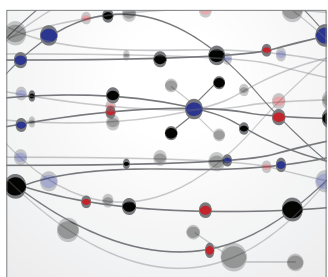

The Scientific World Journal
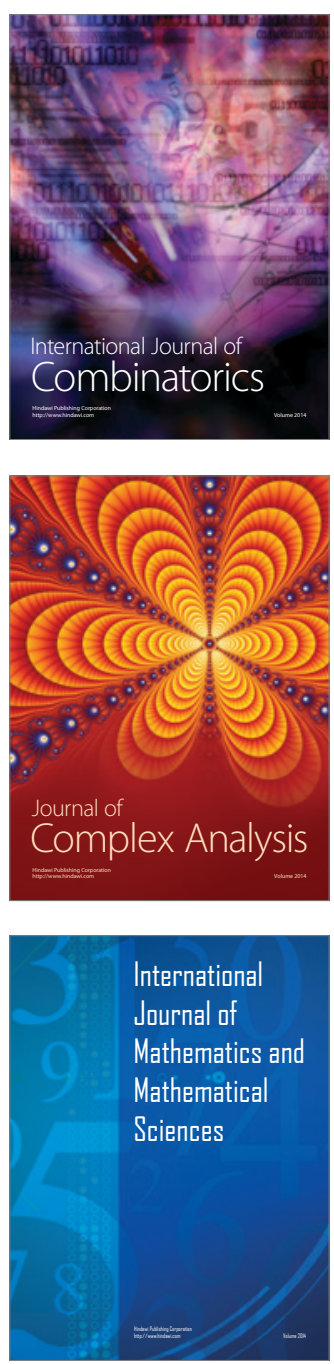
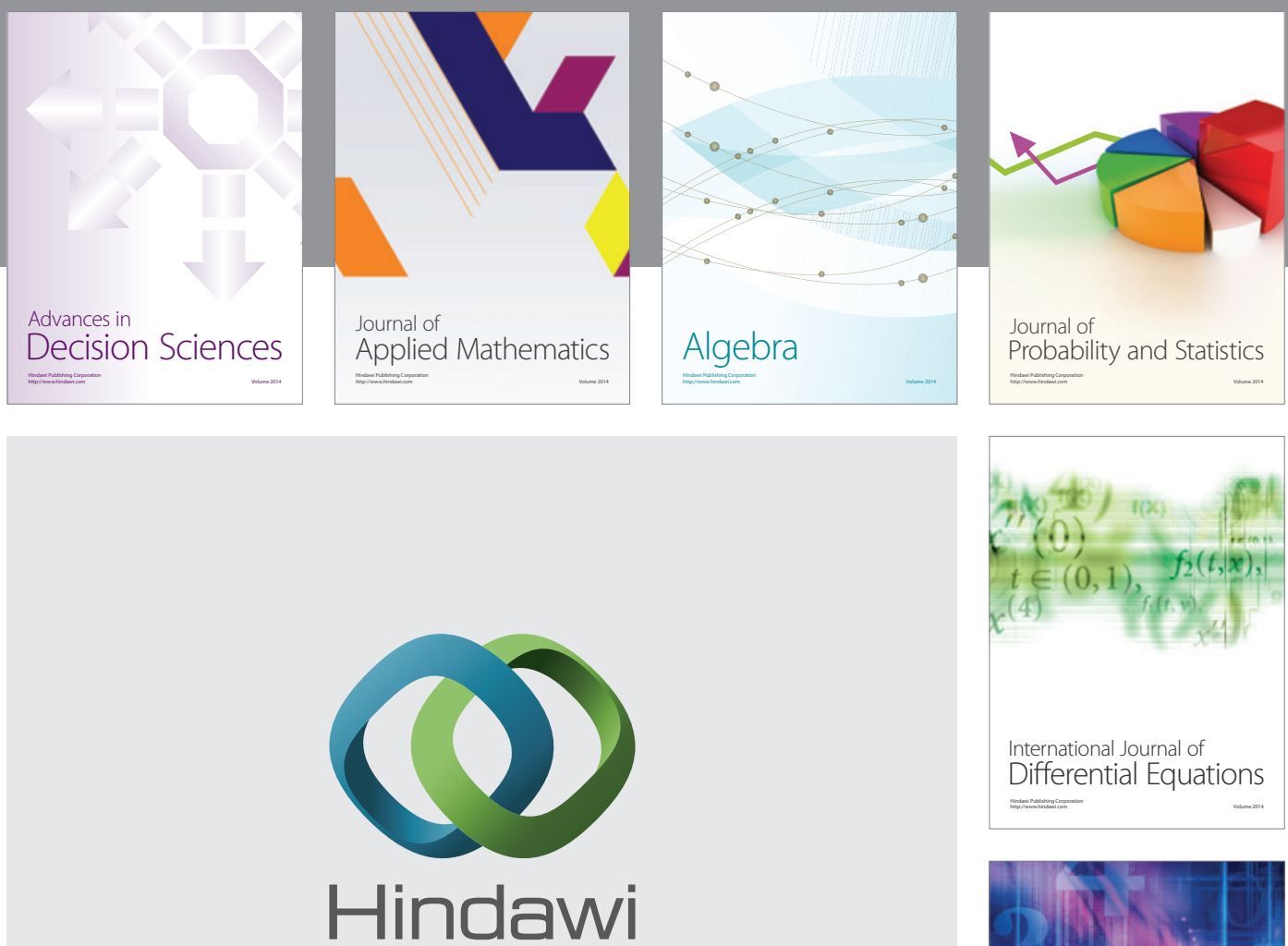

Submit your manuscripts at http://www.hindawi.com
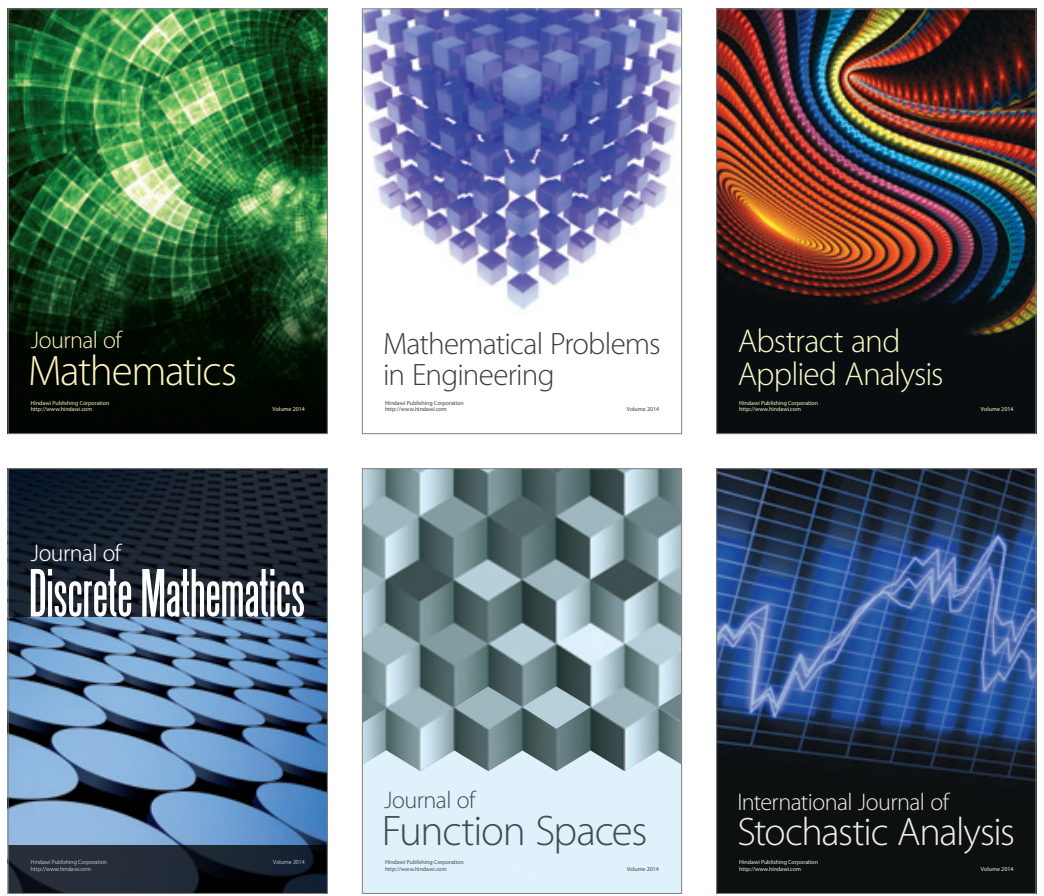

Journal of

Function Spaces

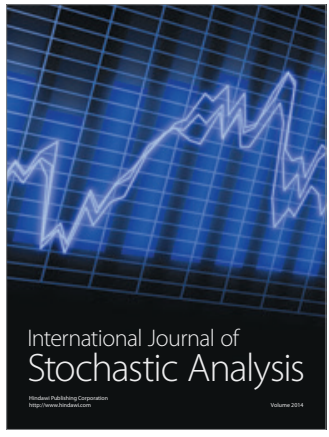

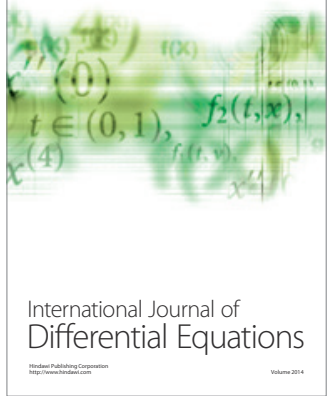
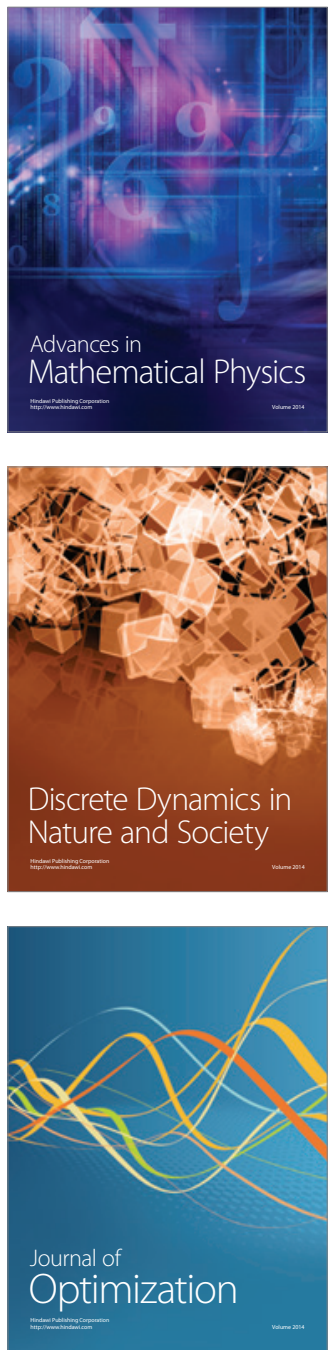